\title{
HOTCAP: a new software package for high-speed oscilloscope- based three-dimensional bunch charge and position measurement
}

\author{
Xing-Yi Xu ${ }^{1,2}$ (1) $\cdot$ Yong-Bin Leng ${ }^{1,2,3} \cdot$ Bo Gao $^{3} \cdot$ Yi-Mei Zhou ${ }^{3} \cdot$ Shan-Shan Cao ${ }^{3} \cdot$ \\ Jian Chen ${ }^{3}$ Fang-Zhou Chen ${ }^{3}$
}

Received: 14 September 2021/Revised: 19 October 2021 / Accepted: 21 October 2021/Published online: 24 November 2021 (C) The Author(s) 2021

\begin{abstract}
A software package to be used in high-speed oscilloscope-based three-dimensional bunch-by-bunch charge and position measurement is presented. The software package takes the pick-up electrode signal waveform recorded by the high-speed oscilloscope as input, and it calculates and outputs the bunch-by-bunch charge and position. In addition to enabling a three-dimensional observation of the motion of each passing bunch on all beam position monitor pick-up electrodes, it offers many additional features such as injection analysis, bunch response function reconstruction, and turn-by-turn beam analysis. The software package has an easy-to-understand graphical user interface and convenient interactive operation, which has been verified on the Windows 10 system.
\end{abstract}

Keywords Bunch-by-bunch measurement · HOTCAP software package $\cdot$ Data analysis $\cdot$ High-speed oscilloscope

This work was supported by the Ten Thousand Talent Program and National Natural Science Foundation of China (No. 11575282) and the Ten Thousand Talent Program and Chinese Academy of Sciences Key Technology Talent Program.

Yong-Bin Leng

lengyongbin@sinap.ac.cn

1 Shanghai Institute of Applied Physics, Chinese Academy of Sciences, Shanghai 201800, China

2 University of Chinese Academy of Sciences, Beijing 100049, China

3 Shanghai Advanced Research Institute, Chinese Academy of Sciences, Shanghai 201204, China

\section{Introduction}

New-generation synchrotron radiation light sources have the following basic characteristics: ultra-low beam emittance (a few nmrad or even tens of pmrad), ultra-high beam stability (with orbital feedback control accuracy being mostly at the micrometer or submicrometer level), high average beam current $(>300 \mathrm{~mA})$, a small-aperture beam vacuum pipe $(<30 \mathrm{~mm}$ in diameter), a large number of vacuum inserts, top-up operation mode, small dynamic aperture, and high time resolution experimental ability (of the order of picoseconds) [1, 2]. The above-mentioned characteristics make how to minimize instability a key technological problem to solve. New-generation light sources have higher requirements for beam stability. Accurate beam diagnosis technology guarantees smooth operation of synchrotron radiation facilities. Bunch-bybunch measurement technology is a popular research direction in the field of beam diagnosis. Using bunch-bybunch measurement technology enables accurate beam diagnosis for each bunch rather than just for the average status of all bunches. This is beneficial for the operating staff to better understand the operating status of the device, facilitating targeted optimization to ensure that the synchrotron radiation device has a better light supply quality [3-5].

Developing a set of bunch-by-bunch signal processing algorithms and establishing a bunch-by-bunch parameter measurement system with high resolution and high refresh rate are crucial. In the early days, limited by electronics technology, beam measurement generally performed on the storage ring mainly included beam closed-track measurement [6] and turn-by-turn beam measurement [7, 8]. In recent years, with the development of electronic technology and the need for accelerator machine research, high- 
precision bunch-by-bunch measurement has become important in accelerator research around the world [9-11].

Bunch-by-bunch measurement mainly includes transverse position measurements [12-14], longitudinal phase measurements [15-17], and charge measurements [18-20], as well as beam size measurement and emittance [21-23]. However, position measurement is the most important and widely used method. The commonly used measurement methods mainly include two categories: phase-locked sampling with an external clock and random phase sampling with a constant frequency. The phase-locked sampling method with an external clock is usually based on a commercial bunch-by-bunch feedback system or a highspeed acquisition board. The random phase sampling method with a constant frequency is usually based on a digital oscilloscope.

Compared with high-speed acquisition boards and commercial feedback systems, digital oscilloscopes usually have a higher bandwidth and sampling rate. The random phase sampling method can be more convenient for performing secondary development to further study the bunches at different accelerators [24-26]. This makes it possible to build a bunch-by-bunch system that is common to all major accelerators worldwide. Therefore, it is advantageous to use a digital oscilloscope as a signal acquisition device for bunch-by-bunch measurement [27-29].

The sampling mode of a digital oscilloscope is usually asynchronous; that is, the signal acquisition does not use the accelerator's radio-frequency (RF) signal as an external clock. Signal acquisition is completely triggered by an internal clock. The advantage of this is that all acquisition results and further processing results are based on the absolute time when the accuracy of the internal clock is much better than the measurement accuracy. However, the high sampling rate and the non-phase-locked sampling mode result in a large amount of input data and complex digital signal processing. Currently, there are no comprehensive data processing algorithms or corresponding data processing software packages that are widely used in highspeed oscilloscope-based three-dimensional (3D) bunch charge and position measurements.

Based on the cross-correlation method, the Shanghai Synchrotron Radiation Facility (SSRF) Beam Instrument Group has studied a set of signal processing algorithms for high-speed oscilloscope-based 3D bunch charge and position measurement and released a visualization software package called HOTCAP. Each step of the parameter setting, data processing, and result reporting can be fulfilled using a mouse click.

The theoretical basis for this package is briefly described in Sect. 2. The main features and capabilities of HOTCAP are presented in Sect. 3. To further verify its usefulness, test experiments at the SSRF and at the Hefei Light Source (HLS) are presented in Sect. 4. Section 5 provides a summary of the results.

\section{Theoretical basis}

The button beam position monitor (BPM) is usually used to measure the position of the beam. There were four button-type electrodes within the BPM. The signal obtained from the button-type electrode contains almost all the bunch-by-bunch information.

When a bunch passes through the button electrode, the electrical signal is drawn from the electrode by the feedthrough, which contains almost all the parameter information of the bunch. The electrical signal collected by the collection device can be expressed as

$$
\begin{aligned}
V(t) & =I(t) \cdot Z \\
& =\frac{-Z}{\beta c} \cdot \frac{t-t_{0}}{\alpha^{2}} \cdot I_{0}(t) \cdot f(\text { area }) \cdot F(\delta, \theta),
\end{aligned}
$$

where $I(t)$ is the current intensity of the resistor. A bunch with a longitudinal Gaussian distribution can be expressed in the time domain as $I_{0}(t)[30,31]$.

It can be seen that the collected voltage signal is related to the transverse position $(F(\delta, \theta))$, longitudinal position (longitudinal phase $\left.t_{0}\right)$, and charge $(e \cdot N)$ of the bunch. Unfortunately, the actual collected signal is not completely in accordance with the theoretical derivation, and the limited bandwidth of the feedthrough introduces time delays. In addition, the bandwidth limitation of the acquisition device also changes the signal waveform in the time domain.

Therefore, it is theoretically feasible to build a bunchby-bunch $3 \mathrm{D}$ charge system by using the button BPM, which is common in almost all accelerator storage rings.

\section{Main features and capabilities}

In this system, button BPMs are used as the bunch signal pick-up. A high-speed broadband oscilloscope was chosen to record the signals from the four button electrodes of the BPMs. These signals can be directly read into the memory using the software package HOTCAP as input to HOTCAP and processed to obtain various physical parameters that characterize the beam current and machine state. The system framework is shown in Fig. 1.

The software package was developed based on Python, and the visual interface was based on PyQT5. To improve the operation speed, NUMBA was used as the acceleration scheme of the compiled script in functions with a large 


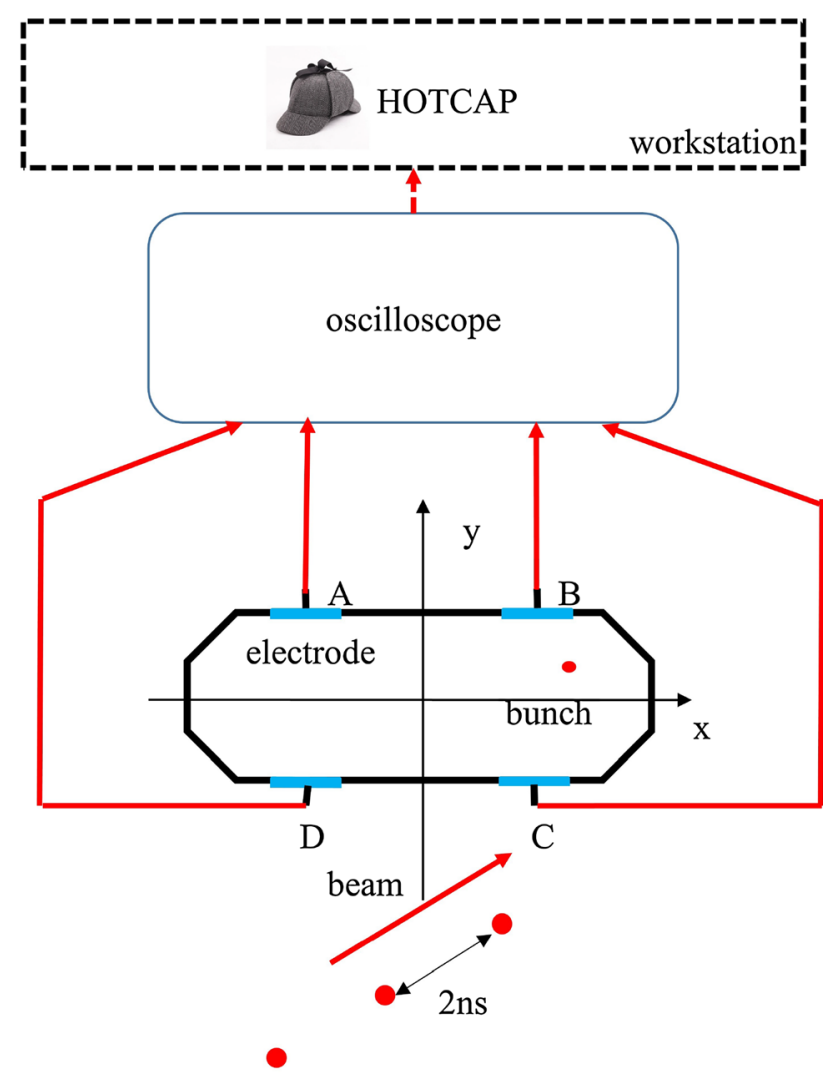

Fig. 1 BPM measurement setup of the bunch-by-bunch measurement system

amount of calculations. The software package was divided into three parts: the user interface (UI) module, the calculation module, and input-output (IO) module. The calculation module was run in a separate child thread.

The software package performs bunch-by-bunch 3D parameter extraction, bunch charge extraction, refilled charge parameter extraction, and analysis of the BPM local wake field. The parameter calculation function for this part was realized in the calculation module. The IO module acts as the data interactive interface between the software package and MATLAB, which reads the original oscilloscope file and saves the calculation results into the corresponding format output file. The UI module serves as the console for the user. Although it reads the machine parameters provided by the user as the necessary parameters for calculation, it shows the calculation results to the user in different forms (e.g., bunch-by-bunch-by-turn, filling analysis, etc.). A simplified schema of the system is presented in Fig. 2.

\subsection{UI module}

The user's interaction with HOTCAP is entirely based on an interactive front-end interface. The interface was built with Qt Designer, and its underlying framework was developed based on PyQT5. At present, all user operations can be completed by clicking the mouse. The user must select the data file to be calculated in the console and select the calculation method. By inputting the necessary machine parameters and calculation mode through the interface, HOTCAP sends the data to the calculation module for the corresponding calculation. After the calculation is completed, the calculation results can be displayed in different forms in the UI (bunch-by-bunch display, turn-by-turn display, and refilled charge movement pattern display).

\subsection{IO module}

The IO module realizes the storage of calculation results and data interaction between the software package and the workstation. Currently, HOTCAP's input and output file format is mat. The mat file can be viewed using MATLAB. Such files usually exist in MATLAB MAT-file format. The MAT file extensions are primarily grouped under the data file category. In less common applications, they can also be uncommon files or 3D image files. MAT files have already been supported on Windows, Mac, and Linux platforms. They are compatible with desktop computers and mobile computers. HOTCAP is compatible with $-v 7$ and lower versions of mat-type files.

The package accepts two or four channels of oscilloscope data, which can be collected from two pairs of electrodes in one direction or from four electrodes in two directions. The current IO module requires these data to be stored as a mat file with variable names of BPM1-BPM4. The calculated results can be stored in the workstation in mat form; these results include but are not limited to the 3D position and charge of all bunches, the response function, the current RF, and the local wake field.

In addition, the IO module also undertakes the function of storing the calculation results. When the calculation module has completed the calculation of one set of data, the results are stored in the IO module, freeing the calculation module to calculate the next set of data. The stored calculation results can be displayed on the interface in the form of line charts, bar charts, etc.

\subsection{Calculation module}

The calculation module is the core module of the HOTCAP software package. It is responsible for almost all signal preprocessing and bunch parameter extraction functions. The calculation module is composed of a main calculation function and 17 subfunctions.

The following section introduces the calculation process and the core algorithm of the calculation module in detail. 
Fig. 2 Architecture of the software package

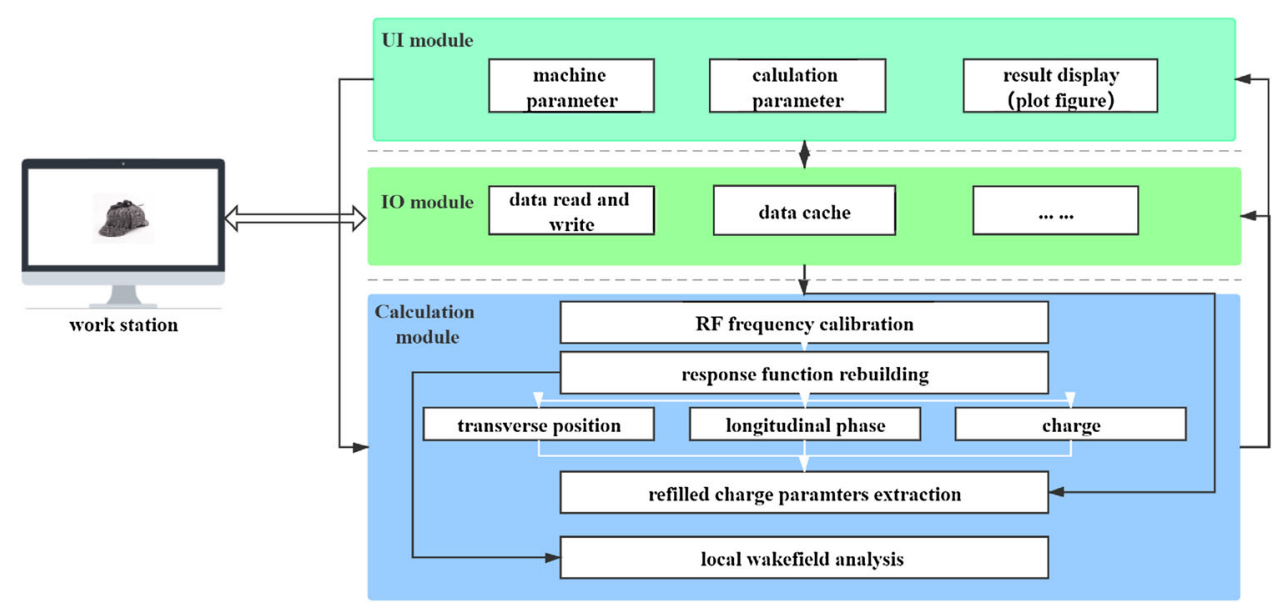

The calculation process is shown in Fig. 3. The primary core algorithms are used for longitudinal phase extraction based on cross-correlation, response function reconstruction based on software resampling, and refilled bunch signal extraction [32].

Fig. 3 Algorithm flowchart for the calculation module

\subsubsection{Preprocess}

Data preprocessing mainly includes normalization, DC compensation, signal initial peak finding, and RF coarse and fine adjustments.

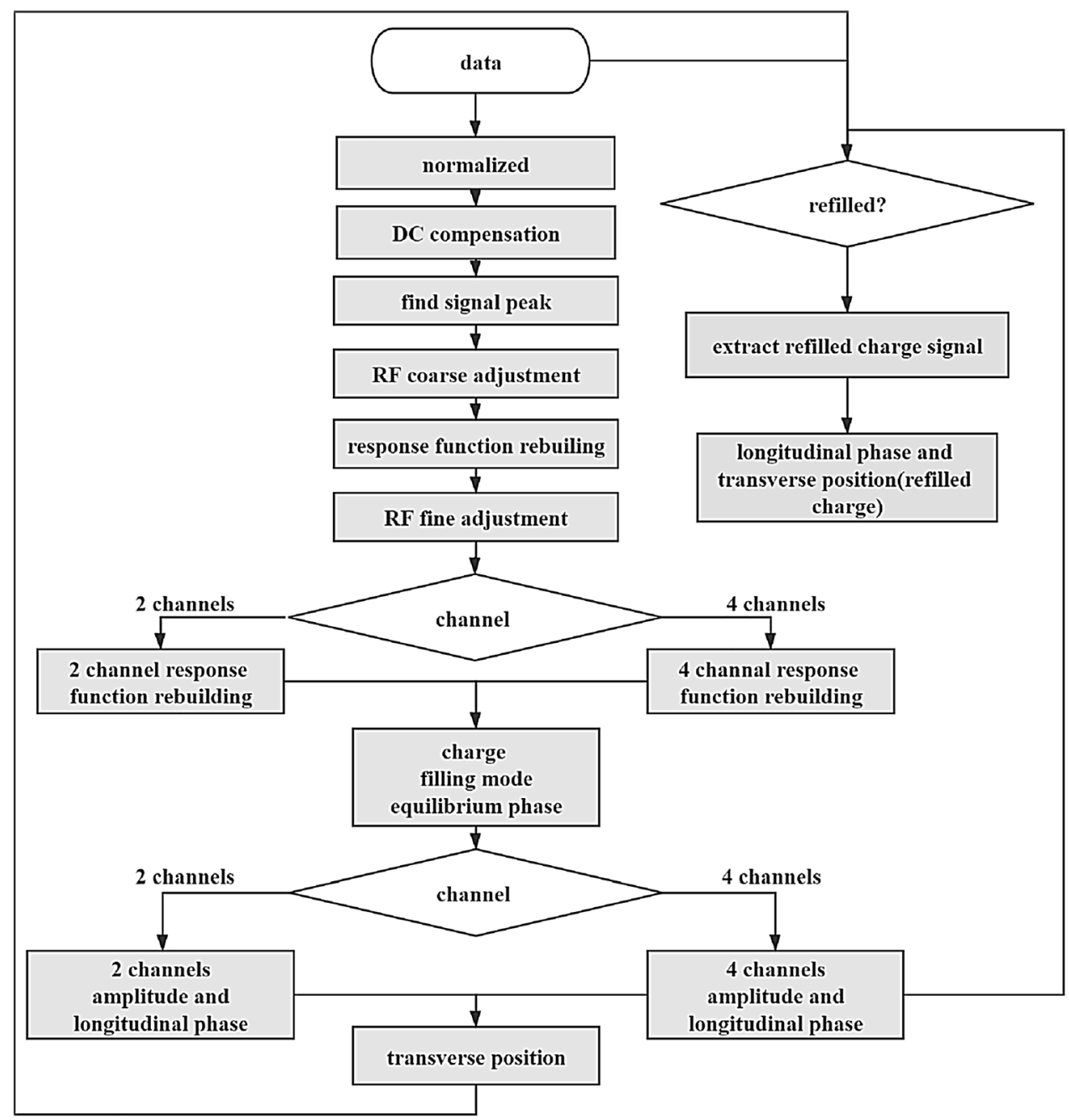


Normalization eliminates the influence of different sampling equipment and normalizes the amplitude of the signal to a fixed range. The DC compensation function removes the $\mathrm{DC}$ bias caused by the acquisition equipment by recording long-term statistics of the value at the null signal (empty bucket). The function of the signal initial peak finding function is to find the peak value of the first bunch at the head of the first bunch train, which is used as the starting point for signal processing.

RF adjustment is the most important part of data preprocessing. Although the RF for a storage ring is usually fixed, in reality, it constantly changes slightly. This RF shift, though small, is very serious for precise 3D bunchby-bunch measurements. Therefore, it is necessary to adjust the RF in real time according to the bunch signal.

The RF adjustment includes two steps: coarse and fine adjustments. During coarse adjustment, the peak point of the signal collected during each turn of the arbitrarily selected probe bunch is used as the feature point, and the time interval between the feature points is calculated. Using the time interval, the RF is then adjusted coarsely. The fine adjustment of the RF is more complicated, and the longitudinal phase of the probe bunch needs to be calculated first. The standard of the fine adjustment is that the equilibrium value of the longitudinal phase remains unchanged. The calculation method for the longitudinal phase is introduced in the following subsections.

\subsubsection{Response function reconstruction}

The response function described in this section refers to the shape of the signal pulse visible to the acquisition device when the bunch passes through the electrode of the BPM, that is, the response of the electrode and the acquisition device to the bunch.

The reconstruction of the response function recovers from the signal the shape of the signal of each bunch, that is, $V(t)$, with a particular bandwidth. The signal excited by a bunch passing through the BPM collected by a broadband device is very short-lived ( $\sim 1 \mathrm{~ns}$ at the SSRF), which means that the acquisition device can only collect a few sampling points. The signal actually has several points on the continuous smooth response function. However, what is needed is a continuous and smooth response function with a higher sampling rate. The reconstruction response function uses multiturn data to splice a response function with a high sampling rate.

For almost all storage rings, the sampling frequency $f$ of the oscilloscope must adhere to the following formula:

$f \neq m f_{\mathrm{c}}$,

where $m$ is a positive integer and $f_{\mathrm{c}}$ is the cyclotron revolution frequency. When the bunch passes through the BPM again, the acquired signal point will not be in the same position as the response function last time. $t_{0}$ can be obtained when the bunch passes the BPM every turn because $f_{\mathrm{c}}$ and $f$ are known. Based on removing the initial phase $t_{0}$, through the splicing of multiturn sampling data, a high sampling rate response function can be constructed.

Owing to the signal noise and oscillation of the transverse position of the bunch, the high sampling rate response function constructed by multiturn splicing is not very smooth. From the perspective of the frequency domain, the splicing signal is mixed with high-frequency noise. From the perspective of the time domain, the line of the spliced response function will be thick. The real response function is the most probable position of the response function obtained by direct splicing. Therefore, this computing module achieves the effect of low-pass filtering through window smoothing to obtain the best response function. A random example was selected to show the process and effect of the reconstruction (shown in Fig. 4).

In HOTCAP's built-in module, the equivalent sampling rate of the reconstructed response function is $100 \mathrm{THz}$; that is, the interval between adjacent points is $0.1 \mathrm{ps}$. If a higher measurement accuracy (of $<0.1 \mathrm{ps)}$ is required, the equivalent sampling rate will need to be increased. This part allows parameters to be adjusted in the secondary development of the HOTCAP package. Because the influence of the measurement error of common sampling devices is often $>0.1 \mathrm{ps}$, the default value of the equivalent sampling rate is set to $100 \mathrm{THz}$, which helps to reduce the amount of computation.

Because the response functions of different bunches collected by different electrodes are different, they are all reconstructed independently. In addition, because the response function is obtained by the splicing of multiple

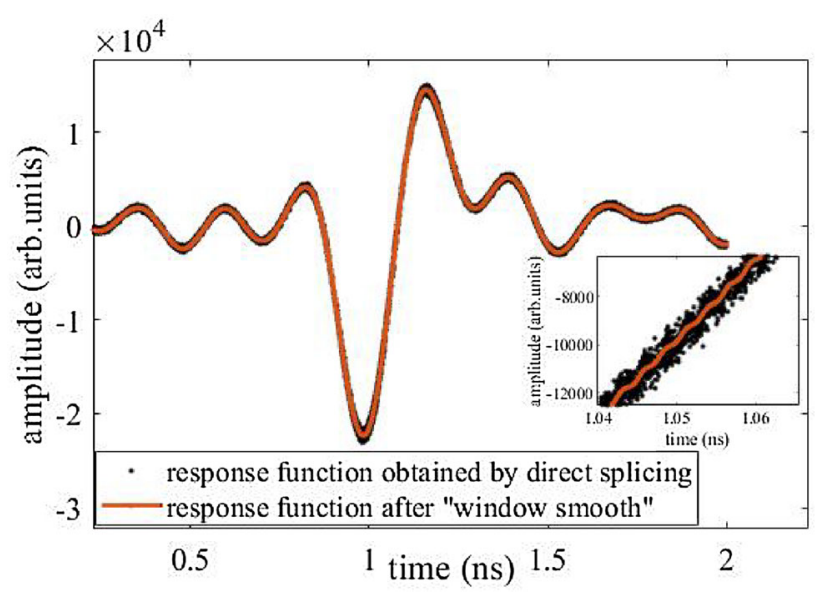

Fig. 4 Response function obtained by direct splicing (black) and the standard response function after window smoothing (red) of a typical bunch. (Color figure online) 
measurement results, this algorithm acts on the premise that the response function of the bunch does not change dramatically in a short time. This requires that the bunch length and bunch charge do not change dramatically in a short time $(\sim 1 \mathrm{~ms})$. Most stable synchrotron radiation devices meet this requirement. If this condition is not met in a special machine status, the shape of the response function with different bunch lengths needs to be constructed and calibrated in advance. The HOTCAP package does not currently support this extremely special machine status, requiring secondary development of the package by the user.

\subsubsection{Phase and position extracting method}

The longitudinal phase and transverse amplitude extraction are the most important parameters in the HOTCAP package. The longitudinal phase extraction is based on the sliding cross-correlation method. The transverse position is obtained by the difference ratio sum of the signal amplitudes of the four electrodes. The amplitude of the signal is then obtained by a linear fitting.

As shown in Fig. 5, the blue line represents the response function of the signal when a bunch passes through an electrode. The response function represented by the blue line is composed of a sampling point in thousands of turns. The yellow line represents the temporary response function excited when the bunch passes through the electrode in the $N$ th turn. The relationship between the blue standard response function and the yellow temporary response function in the $N$ th turn can be expressed as

$$
\operatorname{Func}_{N}(t)=\operatorname{amp}_{N} \cdot \operatorname{Func}\left(t+\varphi_{N}\right),
$$

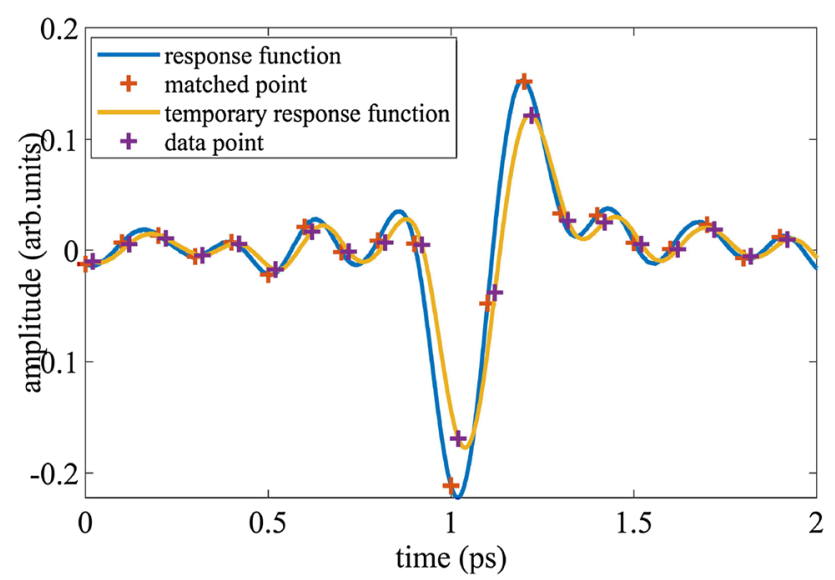

Fig. 5 Schematic of longitudinal phase extraction based on the sliding cross-correlation method. The method is sliding to minimize the angle between the response vector (matched point array) and data vector (data point array). (Color figure online) where Func is the response function with a blue line, $\mathrm{amp}_{N}$ is the relative amplitude of the $N$ th turn, and $\varphi_{N}$ is the relative longitudinal phase in the $N$ th turn. These are very important for the calculation of the transverse and longitudinal phases.

In fact, the temporary response function represented by the yellow line is not available because the sampling rate of the acquisition devices is insufficient. We obtain a number of discrete sample points (represented by purple + signs). The standard response function represented by the blue line is obtained with the response function reconstruction method presented in the previous section. Therefore, the main function of the algorithm module described in this section is to calculate the phase and amplitude with the response function represented by the blue line and the data points acquired in each turn represented by the purple + signs as input data.

The data points acquired in each turn are actually a continuous and equally spaced sampling of the temporary response function in this turn, which can be expressed as

$$
\left\{\mathbf{d}_{N}^{n}\right\}=\operatorname{Func}_{N}\left(t_{0}+\frac{n}{f}\right) \quad(n=1,2,3, \ldots),
$$

where $\mathbf{d}_{N}^{n}$ is the data point array in the Nth turn, $n$ is the sampling index, and $t_{0}$ is the initial sampling phase, which can be obtained using $f$ and $f_{\mathrm{c}}$. Using the sliding crosscorrelation method, the relative phase is obtained by the sampling array $\left\{\mathbf{d}_{N}^{n}\right\}$ and the standard response function (Func). The operation method is described in detail in the following section.

The first step is to resample the standard response function using the oscilloscope sampling interval as the sampling interval. The sliding in cross-correlation means that software resampling is repeated several times in succession with different initial sampling phases $(\varphi(i))$. The resampling can be expressed as

$$
\left\{\mathbf{L U T}^{n, i}\right\}=\operatorname{Func}\left(t_{i}+\frac{n}{f}\right) \quad(n=1,2,3, \ldots),
$$

where $\mathbf{L U T}^{n, i}$ is the resampling data point array with the initial sampling phase $\left(t_{i}\right)$. From Eqs. (3)-(5), if $t_{0}+\varphi_{N}$ is equal to $t_{i}$,

$$
\left\{\mathbf{d}_{N}^{n}\right\}=\operatorname{amp}_{N} \cdot\left\{\mathbf{L} \mathbf{U} \mathbf{T}^{n, i}\right\} \quad(n=1,2,3, \ldots) .
$$

With the Pearson correlation coefficient chosen as the correlation coefficient, the cross-correlation coefficient can be expressed as

$$
\text { correlation_coefficient }=\frac{\left\{\mathbf{d}_{N}^{n}\right\} \cdot \mathbf{L} \mathbf{U} \mathbf{T}^{n, i}}{\left|\left\{\mathbf{d}_{N}^{n}\right\}\right| \cdot\left|\mathbf{L} \mathbf{U} \mathbf{T}^{n, i}\right|} .
$$

Mathematically, this coefficient represents the cosine of the angle between two vectors, ranging from -1 to 1 . If and 
only if Eq. (6), the correlation coefficient is equal to one $\left(\mathrm{amp}_{N}>0\right)$. In this case, $\varphi_{N}$ can be obtained from

$$
\varphi_{N}=t_{i}-t_{0}
$$

Simultaneously, the relative amplitude $\left(\mathrm{amp}_{N}\right)$ in the $N$ th turn can be obtained by a linear fitting:

$$
\operatorname{amp}_{N}=\frac{\left\{\mathbf{d}_{N}^{n}\right\}}{\left\{\mathbf{L} \mathbf{U T}^{n, i}\right\}} .
$$

Thus far, the phase $\left(\varphi_{N}\right)$ and relative amplitude $\left(\mathrm{amp}_{N}\right)$ in Eq. (3) are calculated. Clearly, $\varphi_{N}$ is the longitudinal phase. The absolute amplitude with different electrodes (A, $\mathrm{B}, \mathrm{C}$, or $\mathrm{D})$ is the relative amplitude multiplied by the amplitude of the corresponding standard function. The peak-to-peak value of the standard response function is taken as the amplitude of the standard response function. The bunch-by-bunch transverse position $(x, y)$ is expressed as follows:

$$
\begin{aligned}
& x=k_{x} \cdot \frac{a m p_{A}-a m p_{B}-a m p_{C}+a m p_{D}}{a m p_{A}+a m p_{B}+a m p_{C}+a m p_{D}}, \\
& y=k_{y} \cdot \frac{a m p_{A}+a m p_{B}-a m p_{C}-a m p_{D}}{a m p_{A}+a m p_{B}+a m p_{C}+a m p_{D}},
\end{aligned}
$$

where $k_{x}$ and $k_{y}$ are constants. Eq. (10) is valid only when the beam is at the center of the vacuum chamber. The offset beam includes a systematic error. For most synchrotron radiation facilities, the beam is very close to the center of the vacuum chamber, so this algorithm can meet the accuracy requirements. It should be implemented to include a polynomial fit to reproduce the monitor chamber structure if the beam is far from the center of the vacuum chamber [33, 34]. This is not the core part of the software. If the user has a special machine condition, a nonlinear correction can be added by modifying the HOTCAP code.

\subsubsection{Refilled charge signal extracting method}

The top-up operation mode requires frequent injection to maintain a constant high current. This top-up injection process is a perturbation to the storage ring, which deflects the beam far away from the equilibrium point. The refilled bunch contains stored and refilled charges. The signal collected by the BPMs is a combination of stored and refilled charges. During the injection process, the $3 \mathrm{D}$ movement of the newly refilled charge carries different information from the 3D movement of the stored charge [24].

The HOTCAP software package contains an algorithm module that extracts the refilled charge signal from the refilled bunch signal, enabling the evolution of the $3 \mathrm{D}$ position of the refilled charge to be further extracted. The principles of this module are briefly described in this section.

The refilled bunch consists of the stored charge and refilled charge that has just been injected. For a linear measurement system, the acquired signal of the refilled bunch can be expressed as

$$
\operatorname{signal}(n)=\operatorname{signal}_{\mathrm{s}}(n)+\operatorname{signal}_{\mathrm{r}}(n),
$$

which is the sum of the refilled charge signal $\left(\operatorname{signal}_{\mathrm{r}}(n)\right)$ and the stored charge signal $\left(\operatorname{signal}_{\mathrm{s}}(n)\right)$. To obtain the refilled charge signal, the stored charge signal needs to be subtracted from the acquired signal. However, it is very difficult to obtain the signal of the stored charge because it is mixed in the sum signal.

The algorithm used in the HOTCAP software package is implemented as follows: The first step entails finding the injection transient and refilled bunch index by the sudden change in the charge. In the second step, the 3D position of the stored charge in the refilled bunch is obtained indirectly using the common mode of the 3D position of all bunches. The third step involves reconstructing the stored charge signal according to the $3 \mathrm{D}$ position of the stored charge. In the last step, the reconstructed stored charge signal is subtracted from the acquired signal to obtain the refilled charge signal.

In most synchrotron radiation facilities, adjacent bunches have similar 3D positions. Therefore, the average positions of several stored bunches (without refilled charge) near the refilled bunch were selected as the stored charge positions in the refilled bunch. For HOTCAP, there are 10 nearby bunches. The specific value should be selected according to the situation of different machines. Because it is necessary to know the charge of the refilled charge and the charge of the stored charge when rebuilding the signal, the response function of the stored charge is constructed in advance before the injection occurs.

\subsubsection{Experiments at the SSRF}

It is worth mentioning that, because the 3D position of bunches is constantly changing, it is necessary to calculate the 3D position of stored bunches at each moment (each turn) in advance. The purpose is to obtain the 3D position of the stored charge in the refilled bunch at each turn. This is very important for refilled charge signal extraction.

\section{Test examples}

To test the reliability of the developed package HOTCAP, experiments were conducted at two different synchrotron radiation facilities: the SSRF and the HLS. 
The SSRF, which consists of a $150-\mathrm{MeV}$ linear accelerator, a $180-\mathrm{m}, 3.5-\mathrm{GeV}$ booster, and a $432-\mathrm{m}, 3.5-\mathrm{GeV}$ storage ring, is a multibunch, high-energy third-generation light source. The harmonic number was 720 at an RF of 499.654 MHz. The parameters of the SSRF are listed in Table 1. To improve the efficiency and quality of the light, a top-up filling pattern was adopted at the end of 2012, which resulted in more frequent beam injections and storage of $\sim 500$ bunches with a 2-ns spacing [10, 35].

Two different high-speed oscilloscopes, one with a sampling rate of $10 \mathrm{GHz}$ and a bandwidth of $4.2 \mathrm{GHz}$ and another with sampling rate of $25 \mathrm{GHz}$ and a bandwidth of $10 \mathrm{GHz}$, were used, respectively, in the experiment. The 3D position evolution of the stationary light supply time and injection transient was studied.

Typical results plotted in this section were processed using the data recorded on July 25, 2020, unless otherwise noted (shown in Fig 6).

The results of typical injection transient record processing are shown in the package interface. In the experiment, the trigger of the oscilloscope was set to capture the injection transient, and the data of four channels (from four electrodes) were imported and loaded into the software package. After the calculation was completed, the results were displayed in four subinterfaces (parameter setting, bunch-by-bunch, turn-by-turn, and injection analysis).

As can be seen from the left figure of the parameter setting subinterface (Fig. 6a), the filling pattern of the SSRF is four trains, each consisting of more than 100 bunches with roughly equal charges. The 3D position, response function, and correlation coefficient of any bunch are shown in the bunch-by-bunch subinterface (Fig. 6b). As can be seen from the horizontal position shown on the upper left, the refilling process occurs around the 800th turn. Subsequently, the stored bunch is pushed far away from the equilibrium point by the impact of the kicker's field and displays a typical damped oscillation. By setting the bunch number, the user can display the information about the bunch that the user wants to study. In the turn-byturn subinterface (Fig. 6c), the weighted average of each bunch is weighted by the charge to obtain the 3D turn-byturn information. The instability caused by the filling

Table 1 SSRF and HLS parameters

\begin{tabular}{lll}
\hline Parameter & SSRF & HLS \\
\hline Energy, $E(\mathrm{GeV})$ & 3.5 & 0.8 \\
Radio frequency, $f_{\mathrm{RF}}(\mathrm{MHz})$ & 499.654 & 204.030 \\
Harmonic number, $h$ & 720 & 45 \\
Cyclotron revolution frequency, $f_{\mathrm{c}}(\mathrm{kHz})$ & 694 & 4534 \\
Bunch length, $\sigma(\mathrm{ps})$ & 18 & 50 \\
\hline
\end{tabular}

process can be clearly seen in the turn-by-turn 3D position. The 3D position of the refilled charge is shown in the injection analysis subinterface (Fig. 6d). After the completion of the refilled charge extraction, the refilled charge with the maximum charge was selected as the calculation object. Its 3D position parameters and charge were calculated and displayed in the subinterface. From the longitudinal phase figure on the upper right, it can be clearly seen that, after injection, the longitudinal phase of the refilled charge exhibits a typical longitudinal damping oscillation, which is consistent with the theory.

The HLS, which consists of a linear accelerator and a 0.8-GeV storage ring, is a multibunch, high-energy light source. The harmonic number is 45 at an RF of 204.03 $\mathrm{MHz}$ [36]. The parameters of the HLS are listed in Table 1. To improve the efficiency and quality of the light, a top-up filling pattern was adopted, which resulted in more frequent beam injections and storage of $\sim 35$ bunches with a spacing of $5 \mathrm{~ns}$. It can be seen that the machine parameters of the HLS and SSRF are quite different, making them suitable choices to verify the good generalization of HOTCAP.

The HLS is a second-generation synchrotron radiation accelerator built in the last century. Compared with the third-generation synchrotron radiation accelerator, the beam 3D position is not stable and the difference between the bunches is very large; that is, the $3 \mathrm{D}$ position of the adjacent bunches is very different. We analyzed the possible reasons for this phenomenon. First, there is a roomtemperature accelerating cavity at the HLS but not a superconducting accelerating cavity. In addition, highorder harmonic cavities may also increase the difference between bunches. We hope to use the HOTCAP software package to study the instability phenomenon and the difference between bunches at the HLS.

HOTCAP supports exporting bunch-by-bunch results in a file. Three typical bunches (a, b, and c) were selected from the 35 bunches stored in the HLS storage ring, and their 3D position and Pearson correlation coefficient evolutionary process are shown in Fig 7. It can be seen that their 3D positions at the same time are different, which proves the feasibility of the bunch-by-bunch measurement; that is, different bunch statuses can be measured simultaneously. In addition, the bunches in the HLS have a longitudinal phase oscillation. The oscillation amplitude was $\sim 100 \mathrm{ps}$, and the oscillation amplitudes of different numbered bunches were different at the same time. We suspect that this phenomenon is caused by the room-temperature accelerating cavity and the high-order harmonic cavity.

It is interesting to note that the amplitude of the horizontal position (red lines in a, b, and c) and the longitudinal phase oscillations (black lines in a, b, and c) are consistent, 
Fig. 6 (Color online) HOTCAP software package result display interfaces. The example data were recorded on July 25, 2020, at the SSRF: a parameter setting subinterface; b bunch-by-bunch subinterface; c turn-by-turn subinterface; d injection analysis subinterface
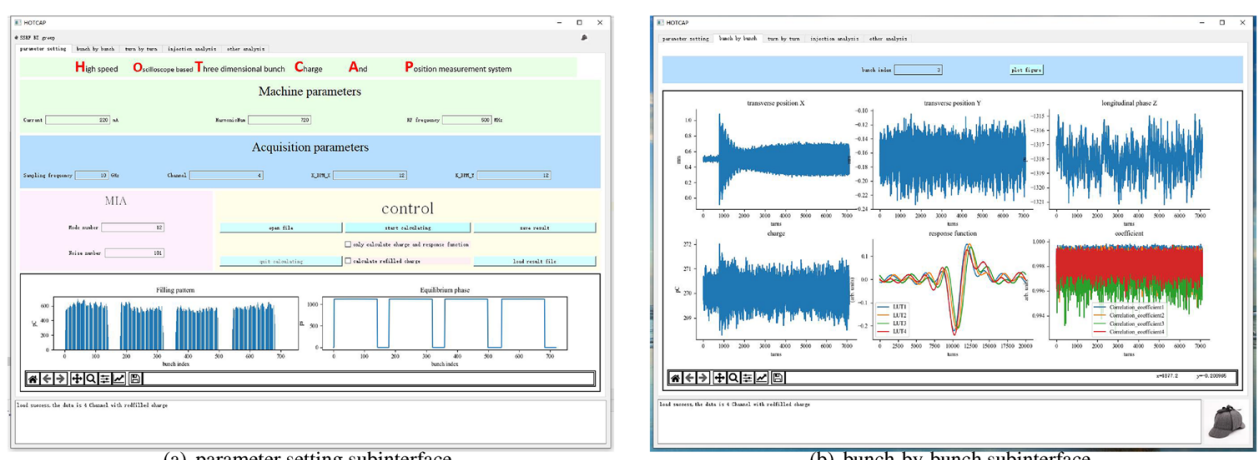

(a) parameter setting subinterface
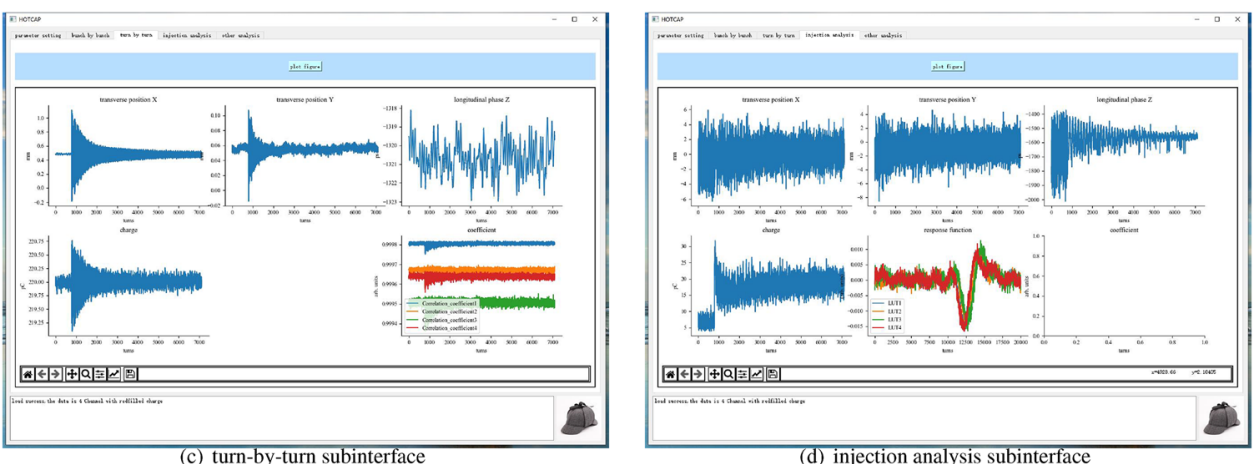

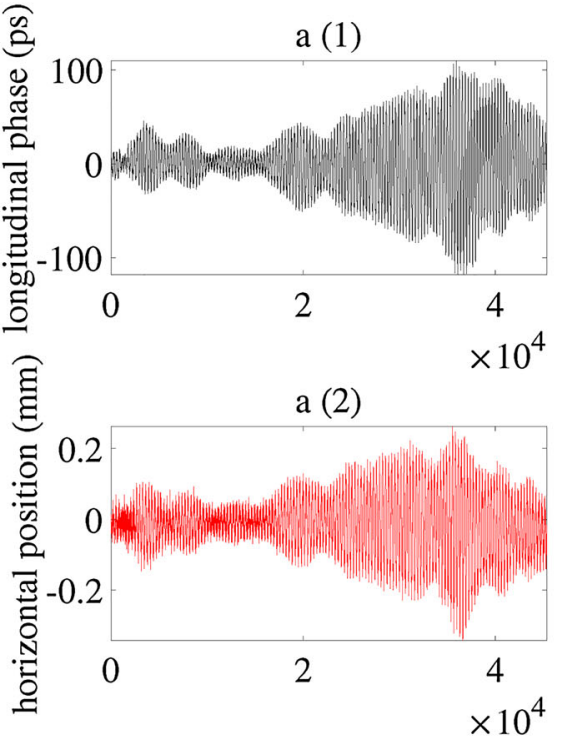

a (3)

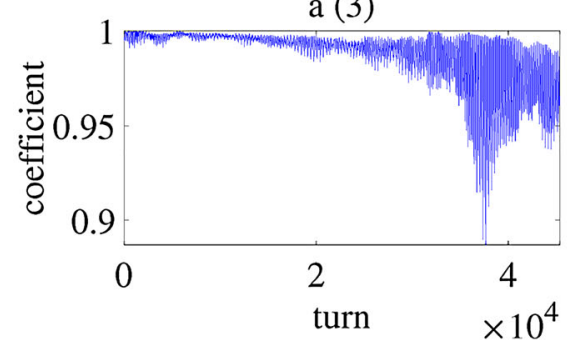

b (1)

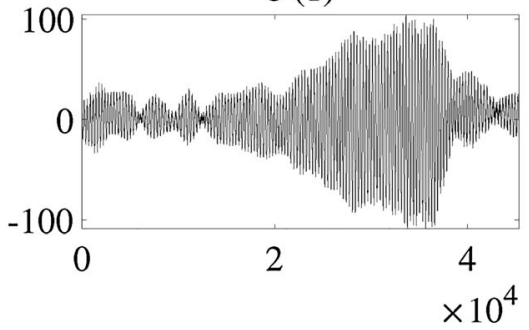

b (2)

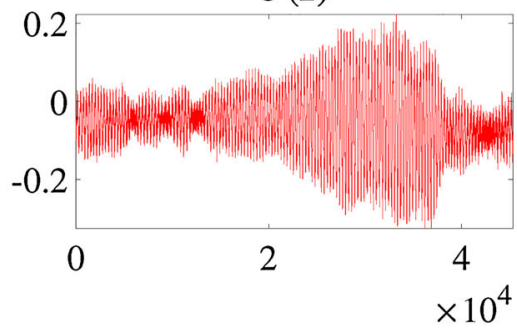

b (3)

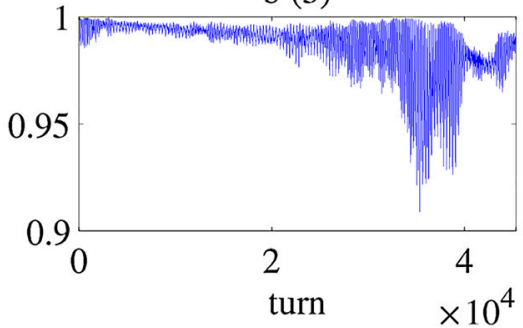

$\mathrm{c}(1)$

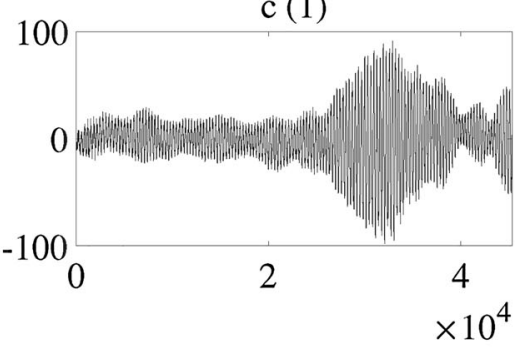

c (2)

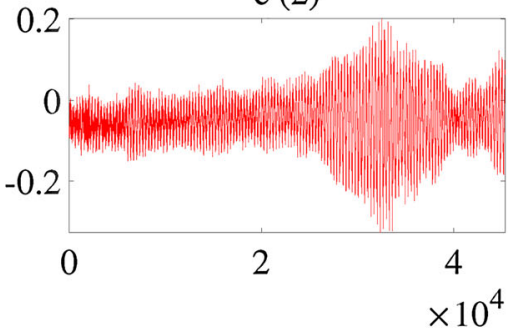

c (3)

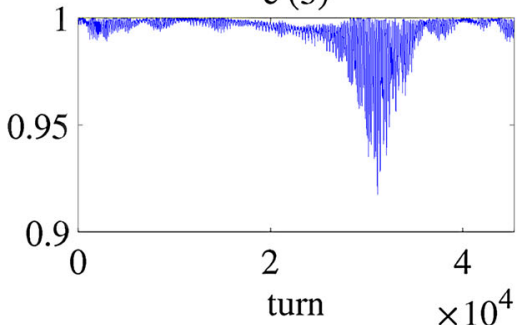

Fig. 7 (Color online) 3D position and Pearson correlation coefficient evolutionary process of three typical adjacent bunches (a-c). The black, red, and blue lines represent the longitudinal phase, horizontal position, and Pearson correlation coefficient, respectively, for different turns 
because under the influence of the dispersion function, the change in the longitudinal phase will be coupled to the change in the horizontal position.

It can be seen from the blue line (bottom figure) of each bunch that the correlation coefficient is no longer stable near 1 but is often $<1$, which indicates that the effect of cross-correlation matching is not perfect. The reason for this problem is that the bunch length of the HLS is not constant but changes greatly in a short time, which leads to a constant change in the response function. HOTCAP uses a fixed response function as matching data, which affects the calculation accuracy. The correlation coefficient decreases (in $\sim 30,000$ turns) when severe instability occurs. This indicates that the bunch length in the HLS storage ring becomes long and short during this time.

\section{Conclusion}

3D bunch-by-bunch position measurements are very important for beam diagnosis. At present, there is no mature online measurement system that can simultaneously measure the bunch-by-bunch 3D position. In addition, the injection transient is an important window for machine research. Many synchrotron radiation devices operate in the top-up mode, which means frequent refilling. At present, there is no mature online system that can strip the refilled charge signal. Therefore, the Beam Instrument Group of the SSRF developed a 3D position measurement package called HOTCAP. HOTCAP can measure the 3D position of each bunch synchronously and strip the refilled charge signal to further analyze the injection transient. In this study, using a signal acquisition device with a digital oscilloscope, we realized an online measurement system that can simultaneously measure the bunch-by-bunch 3D position and charge. Depending on the developed versatile and user-friendly software package, it also offers many advanced features such as injection transient analysis, bunch response function reconstruction, and turn-by-turn beam analysis. This work is expected to be widely used in similar accelerators and can be further improved.

The HOTCAP package is highly versatile and can be applied to most synchrotron radiation facilities. At the same time, the current version of the software package requires that the bunch length in the storage ring be roughly constant. The reason for this requirement is that, if the bunch length changes dramatically, the response function will change accordingly, and the accuracy of the phasematching method based on cross-correlation will be reduced. Given that the bunch length is constant in most third-generation synchrotron radiation facilities, HOTCAP can meet most usage requirements. This problem can be solved in the future by upgrading the algorithm. The specific method would be to scan and calibrate the response functions under different bunch lengths for algorithm matching after actively changing the set value of the bunch length. With the help of this algorithm, HOTCAP would no longer require a constant bunch length but could also calculate the current bunch length in real time from the matching situation.

The current version of HOTCAP is available on GITHUB. ${ }^{1}$ Peers with relevant research needs are free to download and use the package. In the future, we plan to open the source code to the platform so that other peers can redevelop it for specific accelerators.

Acknowledgements We thank the Shanghai Synchrotron Radiation Facility Operation Group and the Hefei Light Source Beam Instrument Group for their help with the experiment.

Open Access This article is licensed under a Creative Commons Attribution 4.0 International License, which permits use, sharing, adaptation, distribution and reproduction in any medium or format, as long as you give appropriate credit to the original author(s) and the source, provide a link to the Creative Commons licence, and indicate if changes were made. The images or other third party material in this article are included in the article's Creative Commons licence, unless indicated otherwise in a credit line to the material. If material is not included in the article's Creative Commons licence and your intended use is not permitted by statutory regulation or exceeds the permitted use, you will need to obtain permission directly from the copyright holder. To view a copy of this licence, visit http://creativecommons. org/licenses/by/4.0/.

Author Contributions All authors contributed to the study conception and design. Material preparation, data collection and analysis were performed by Xing-Yi Xu, Yong-Bin Leng, and Yi-Mei Zhou. The first draft of the manuscript was written by Xing-Yi Xu and all authors commented on previous versions of the manuscript. All authors read and approved the final manuscript.

\section{References}

1. H. Tanaka, Trends and challenges in the future storage ring light sources, in Proceedings of the ICFA Advanced Beam Dynamics Workshop (FLS'18) (JACoW, 2018). http://ir.ihep.ac.cn/handle/ 311005/280654

2. J.J. Liu, X.P. Ma, G.X. Pei et al., Phase-stabilized RF transmission system based on the LLRF controller and optical delay line. Nucl. Sci. Tech. 30(12), 177 (2019). https://doi.org/10.1007/ s41365-019-0697-9

3. Y. Yang, Y.B. Leng, Y.B. Yan et al., Injection performance evaluation for SSRF storage ring. Chin. Phys. C 39, 097003 (2015). https://doi.org/10.1088/1674-1137/39/9/097003

4. L. Duan, Y. Leng, R. Yuan et al., Injection transient study using a two-frequency bunch length measurement system at the SSRF. Nucl. Sci. Tech. 28, 93 (2017). https://doi.org/10.1007/s41365017-0247-2

5. Z. Chen, Y. Yang, Y. Leng et al., Wakefield measurement using principal component analysis on bunch-by-bunch information

\footnotetext{
${ }^{1}$ https://github.com/xuxingyi/HOTCAP.
} 
during transient state of injection in a storage ring. Phys. Rev. Accel. Beams 17, 112803 (2014). https://doi.org/10.1103/Phys RevSTAB.17.112803

6. J.H. Wang, J.Y. Li, Z.P. Liu et al., Modification of BPM system of NSRL electron storage ring and the closed orbit measurement. J. Univ. Sci. Technol. China 28, 732 (1998). (in Chinese)

7. P. Castro, Applications of the 1000-turns orbit measurement system at LEP, in Proceedings of the 1999 Particle Accelerator Conference (Cat. No.99CH36366), vol. 1 (1999), pp. 456-460. https://doi.org/10.1109/PAC.1999.795731

8. Z. Chen, Y. Leng, R. Yuan et al., Study of algorithms of phase advance measurement between BPMs and its application in SSRF. Nucl. Sci. Tech. 22(5), 261-264 (2011). https://doi.org/10. 13538/j.1001-8042/nst.22.261-264

9. H. Chen, J. Chen, B. Gao et al., A fast beam size diagnostic system using high-speed photomultiplier array at SSRF, in Proceedings of IPAC2017, Copenhagen, Denmark (JACoW, Geneva, Switzerland, 2017). https://doi.org/10.18429/JACoW-IPAC2017MOPAB094

10. Y. Zhou, H. Chen, S. Cao et al., Bunch-by-bunch longitudinal phase monitor at SSRF. Nucl. Sci. Tech. 29, 113 (2018). https:// doi.org/10.1007/s41365-018-0445-6

11. X. Xu, Y. Leng, Y. Zhou, Machine learning application in bunch longitudinal phase measurement, in Proceedings of IPAC2019, Melbourne, Australia (JACOW, Melbourne, Australia, 2019), pp. 2625-2628. https://doi.org/10.18429/JACoW-IPAC2019WEPGW064

12. G. Kotzian, D. Valuch, W. Höfle, Sensitivity of the LHC transverse feedback system to intra-bunch motion, in Proceedings of IPAC2017, Copenhagen, Denmark (JACoW, Geneva, Switzerland, 2017). https://doi.org/10.18429/JACoW-IPAC2017-TUPIK093

13. G. Kotzian, Transverse feedback parameter extraction from excitation data, Proceedings of IPAC2017, Copenhagen, Denmark (JACoW, Geneva, Switzerland, 2017). https://doi.org/10. 18429/JACoW-IPAC2017-TUPIK094

14. N.B. Kraljevic, P. Burrows, R. Ramjiawan et al., Optimisation of a high-resolution, low-latency stripline beam position monitor system for use in intraTrain feedback, in Proceedings of IPAC2017, Copenhagen, Denmark (JACoW, Geneva, Switzerland, 2017). https://doi.org/10.18429/JACoW-IPAC2017-TUPIK110

15. K. Scheidt, B. Joly, Upgrade of beam phase monitors for the ESRF injector and storage ring, in Proceedings of IBIC2013 (Oxford, UK, 2013), p. 757. https://accelconf.web.cern.ch/ IBIC2013/papers/wepc33.pdf

16. R.H.A. Farias, L. Lin, A.R.D. Rodrigues et al., Upgrade of beam phase monitors for the ESRF injector and storage ring. Phys. Rev. Accel. Beams 4, 072801 (2001). https://doi.org/10.1103/Phys RevSTAB.4.072801

17. T. Ieiri, K. Akai, H. Fukuma et al., Bunch-by-bunch measurements of the betatron tune and the synchronous phase and their applications to beam dynamics at KEKB. Phys. Rev. Accel. Beams 5, 094402 (2002). https://doi.org/10.1103/PhysRevSTAB.5.094402

18. Y.K. Zhao, B.G. Sun, J.G. Wang et al., Effective improvement of beam lifetime based on RF phase modulation at the HLS-II storage ring. Nucl. Sci. Tech. 32(1), 1 (2021). https://doi.org/10. 1007/s41365-020-00836-0

19. Y. Yang, Y. Leng, Y. Yan et al., Bunch-by-bunch beam position and charge monitor based on broadband scope in SSRF, in Proceedings of IPAC2013, Shanghai, China (2013), pp. 595-597. http://accelconf.web.cern.ch/IPAC2013/papers/mopme054.pdf

20. Z. Chen, Y. Leng, Y. Zou et al., Baseline recovery method to measure bunch charge under low-current mode of SSRF. Nucl. Sci. Tech. 22(5), 261-264 (2011). https://doi.org/10.13538/j. 1001-8042/nst.22.261-264

21. H. Chen, J. Chen, B. Gao et al., Bunch-by-bunch beam size measurement during injection at Shanghai synchrotron radiation facility. Nucl. Sci. Tech. 29, 79 (2018). https://doi.org/10.1007/ s41365-018-0420-2

22. M.W. Wang, Q.Z. Xing, S.X. Zheng et al., Beam position monitors precise phase pickups for beam energy measurement at the Compact Pulsed Hadron Source. Nucl. Sci. Tech. 30(2), 23 (2019). https://doi.org/10.1007/s41365-019-0545-y

23. H.M. Xie, K.W. Gu, Y. Wei et al., A non-invasive ionization profile monitor for transverse beam cooling and orbit oscillation studies in HIRFL-CSR. Nucl. Sci. Tech. 31(4), 40 (2020). https:// doi.org/10.1007/s41365-020-0743-7

24. X. Xu, Y. Zhou, Y. Leng, Machine learning based image processing technology application in bunch longitudinal phase information extraction. Phys. Rev. Accel. Beams 23, 032805 (2020). https://doi.org/10.1103/PhysRevAccelBeams.23.032805

25. X. Xu, Y. Leng, Beam coupling impedance analyze using bunchby-bunch measurement, in Proceedings of IBIC'20, Santos, Brazil (2020), pp. 202-205. https://doi.org/10.18429/JACoWIBIC2020-THAO04

26. X. Xu, Y. Zhou, Y. Leng, New noninvasive measurement method of optics parameters in a storage ring using bunch-by-bunch 3D beam position measurement data. Phys. Rev. Accel. Beams 24, 062802 (2021). https://doi.org/10.1103/PhysRevAccelBeams.24. 062802

27. K.K.T. Nakamura, S. Dat, T. Ohshima, Transverse bunch-bybunch feedback system for the Spring-8 storage ring, in Proceedings of the 9th European Particle Accelerator Conference, Lucerne, 2004 (EPS-AG, Lucerne, 2004), p. 2049. https://accel conf.web.cern.ch/e04/PAPERS/THPLT068.PDF

28. J. Lee, M.H. Chun, G.-J. Kim et al., Bunch-by-bunch position measurement and analysis at PLS-II. J. Synchrotron Radiat. 24, 163 (2017). https://doi.org/10.1107/S1600577516018154

29. F. Chen, L. Lai, Y. Leng et al., Design of a new type of beam charge monitor based on bunch by bunch DAQ system, in Proceedings of IBIC'17, MI, USA, 20-24 August 2017 (JACOW, Geneva, Switzerland, 2018), pp. 284-286. https://doi.org/10. 1103/PhysRevAccelBeams.24.032802

30. R.E. Shafer, Beam position monitoring. AIP Conf. Proc. 249, 601 (1992). https://doi.org/10.1063/1.41980

31. S.R. Smith, Beam position monitor engineering. AIP Conf. Proc. 390, 50 (1997). https://doi.org/10.1063/1.52306

32. X. Xu, Y. Zhou, Y. Leng et al., Bunch-by-bunch three-dimensional position and charge measurement in a storage ring. Phys. Rev. Accel. Beams 24, 032802 (2021). https://doi.org/10.1103/ PhysRevAccelBeams.24.062802

33. G.M. Wang, T. Shaftan, W.X. Cheng, et al., Beam diagnostics using BPM signals from injected and stored beams in a storage ring, in Proceedings of 24th Particle Accelerator Conference (PAC'11), New York, NY, USA, March-April 2011, Paper THP132, pp. 2369-2371. https://accelconf.web.cern.ch/ PAC2011/papers/THP132.PDF

34. W.X. Cheng, B. Bacha, B.N. Kosciuk et al., Performance of NSLS2 button BPM, in Proceedings of 2nd International Beam Instrumentation Conference (IBIC'13), Oxford, UK, September 2013, Paper WEPC09, pp. 678-681. https://accelconf.web.cern. ch/ibic2013/papers/wepc09.pdf

35. H.H. Li, G.M. Liu, W.Z. Zhang et al., Beam dynamics in the SSRF storage ring, in Proceeding of the 1st International Particle Accelerator Conference (JACoW, Kyoto, Japan, 2010), pp. 2591-2593. https://accelconf.web.cern.ch/IPAC10/papers/ wepea045.pdf

36. W. Li, H. Xu, L. Wang et al., The upgraded scheme of Hefei Light Source, in Proceedings of IPAC'10, Kyoto, Japan (JACOW, Melbourne, Australia, 2010). https://doi.org/10.1063/1. 3463324 